\title{
Fibronectin and Hepatocyte Growth Factor Produced by Lung Fibroblasts Augment Migration and Invasion of Malignant Pleural Mesothelioma Cells
}

\author{
NOBUHIRO KANAJI, NOBUYUKI KITA, NORIMITSU KADOWAKI and SHUJI BANDOH \\ Department of Internal Medicine, Division of Hematology, Rheumatology and Respiratory Medicine, \\ Faculty of Medicine, Kagawa University, Kagawa, Japan
}

\begin{abstract}
Background: The interaction between fibroblasts and malignant pleural mesothelioma (MPM) cells is not well understood. Materials and Methods: Lung fibroblasts (HFL1, MRC5 and IMR9O) and MPM cells (H28, H226 and H2052) were cultured under serum-free conditions and the resulting culture media were collected. Migration and invasion of MPM cells were assessed by chemotaxis and Matrigel assays, respectively. Results: Lung fibroblast-derived media enhanced the migration and invasion of the three tested MPM cell lines. Fibronectin and hepatocyte growth factor (HGF) were produced by lung fibroblasts. Exogenous fibronectin and HGF also enhanced the migration and invasion of the three MPM cells, respectively. Neutralizing anti-HGF antibody inhibited the invasion of $\mathrm{H} 28$ cells enhanced by fibroblastderived media. In addition, the production of fibronectin and HGF was stimulated by MPM cell-derived media. Conclusion: The current study provides additional evidence that might contribute to the development of antitumorassociated fibroblast therapeutic strategies.
\end{abstract}

Malignant pleural mesothelioma (MPM) is a relatively uncommon malignancy that arises from the pleura of the lung (1). The development of MPM is associated with exposure to asbestos and MPM incidence is expected to increase dramatically over the next few decades (2). Diagnosis of MPM at an operable stage is rare and prognosis

Correspondence to: Nobuhiro Kanaji, MD, Ph.D., Department of Internal Medicine, Division of Hematology, Rheumatology and Respiratory Medicine, Faculty of Medicine, Kagawa University, 1750-1 Ikenobe, Miki-cho, Kita-gun, Kagawa 761-0793, Japan. Tel: +81 878912145, Fax: +81 878912147, e-mail: kanaji@med.kagawau.ac.jp

Key Words: Mesothelioma, fibroblast, fibronectin, hepatocyte growth factor, cancer-associated fibroblast, tumor-associated fibroblast. of MPM patients is extremely poor (3). Although chemotherapy with cisplatin and pemetrexed results in improved survival rates, median survival time is still not satisfactory (12.1 months vs. 9.3 months in cisplatin alone) (4). In order for better prognosis to be possible for MPM, it is important to investigate and elucidate the mechanisms underlying MPM progression.

Recent evidence has shown that the cancer microenvironment plays a significant role in cancer progression (5). Although most host cells in the cancer microenvironment possess certain cancer-suppressing abilities, the microenvironment changes during malignancy and eventually promotes growth, invasion and metastasis (6). Fibroblasts represent an abundant cell population in the cancer microenvironment (5). These fibroblasts are called carcinoma-associated fibroblasts (CAFs) and acquire a myofibroblast-like phenotype.

The infiltration of fibroblasts called tumor-associated fibroblasts (TAFs) has been detected in clinical specimens from MPM patients (3) and it has been reported that MPM cells stimulate TAFs to produce hepatocyte growth factor (HGF), which promotes MPM cell growth and migration (3). However, besides these observations, little is known regarding the interaction between lung fibroblasts and MPM cells.

To further investigate the role of the interaction between these two cell types in MPM progression, the migration and invasion of MPM cells were assessed in the presence of lung fibroblast-derived culture medium.

\section{Materials and Methods}

Reagents. Human fibronectin was purchased from BD Biosciences (Franklin Lakes, NJ, USA). Recombinant human HGF and transforming growth factor (TGF)- $\beta 1$ were purchased from PeproTech (Rocky Hill, NJ, USA). Anti-human HGF antibody was purchased from R\&D Systems (Minneapolis, MN, USA).

Cell culture and collection of cell-derived medium. Human fetal lung fibroblasts (HFL1, IMR90 and MRC5) and human MPM cell lines 

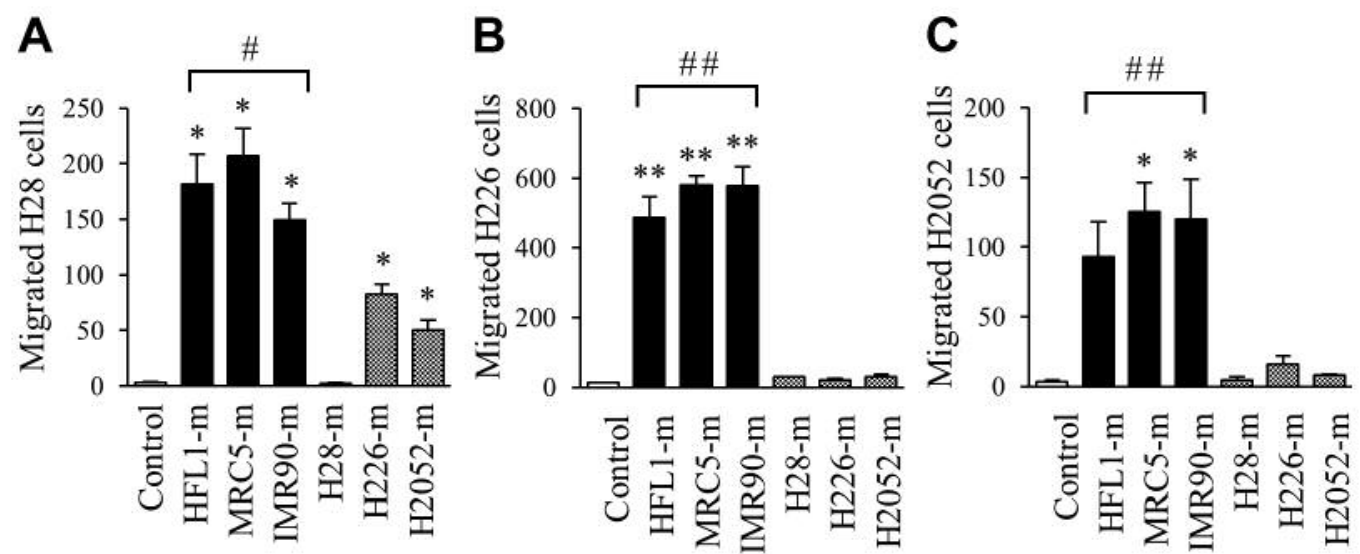

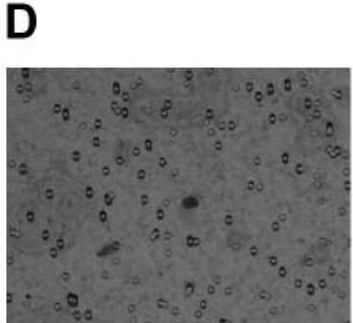

Control

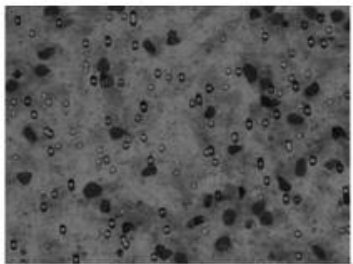

HFL1-m

Figure 1. Malignant pleural mesothelioma (MPM) cell migration in response to lung fibroblast-derived media. Lung fibroblasts and MPM cells were cultured under serum-free conditions for 2 days, after which the culture media were collected. MPM cell migration was assessed by chemotaxis assay. (A) H28 cells; (B) H226 cells; (C) H2052 cells. Data represent migrated cell numbers per five fields (mean \pm SEM of three independent experiments). ${ }^{*} p<0.05,{ }^{* *} p<0.01$ compared to control (fresh medium). ${ }^{\#} p<0.05,{ }^{\# \#} p<0.01$ compared to a total of three MPM cell-derived media. (D) Representative images: $H 28$ cell migration in chemotaxis assay.

(H28, H226 and H2052) were purchased from the American Type Culture Collection (Rockville, MD, USA). The fibroblasts were cultured in Dulbecco's modified Eagle's medium (DMEM) supplemented with $10 \%$ fetal bovine serum (FBS). Fibroblasts were used between the 16th and 20th passages. The MPM cells were cultured in RPMI-1640 medium supplemented with $10 \%$ FBS. To collect MPM cell- and fibroblast-derived medium, subconfluent cells were washed twice with serum-free DMEM before being incubated with serum-free DMEM (2 $\mathrm{ml} / 10^{6}$ cells). After $48 \mathrm{~h}$ of incubation, the medium was collected and centrifuged. The resulting supernatant was then used as either MPM cell- or fibroblast-derived medium and is referred to as, for example, "H28-m" or "HFL1-m" in this study. Each cell-derived medium type was prepared at least three times independently.

Chemotaxis assay. Cell migration was assessed using a 48-well microchemotaxis chamber (Neuroprove, Gaithersburg, MD, USA) as described previously (7). Briefly, fresh serum-free DMEM (control), DMEM containing fibronectin $(10 \mu \mathrm{g} / \mathrm{ml})$ or $\mathrm{HGF}(3 \mathrm{ng} / \mathrm{ml})$ or lung fibroblast-derived media were placed into the bottom wells ( $27 \mu \mathrm{l} /$ well). A non-coated $8-\mu \mathrm{m}$ pore membrane was put on and MPM cells were seeded into the top wells of the chambers $\left(10^{6} \mathrm{cells} / \mathrm{ml}, 50 \mu \mathrm{l} / \mathrm{well}\right)$. Cells were then allowed to migrate through the membrane for 6 hours. Nonmigrated cells were scraped off the membrane and the membrane was then fixed and stained with Diff-Quik (Sysmex, Kobe, Japan). Migrated cell numbers were determined using light microscopy.
In vitro invasion assay. The membrane invasion culture system was used to assess cell invasion (8). Briefly, $4 \times 10^{5}$ MPM cells suspended in RPMI-1640 with $0.5 \%$ FBS were seeded into the inserts of Matrigel-coated invasion chambers (Becton-Dickinson). Fibroblastor MPM cell-derived media containing $0.5 \%$ FBS were placed in the bottom wells. After incubation for 2 days, non-invaded cells were scraped off and the cells invaded through the membrane were fixed and stained with Dif-Quik and microscopically quantified.

Fibronectin and HGF quantification by ELISA. Fibronectin and HGF concentrations in media were determined by Fibronectin (\#KAC2211; Takara Bio Inc., Shiga, Japan) and HGF (\#MK115; Thermo Fisher Scientific, Waltham, MA, USA) ELISA kits, respectively. The kits were used according to the manufacturers' instructions.

Statistical analysis. Each experiment was repeated at least three times. Student's $t$-test was used to compare two-group data. Differences with $p$-values $<0.05$ were considered statistically significant.

\section{Results}

Culture medium derived from lung fibroblasts increases migration and invasion of MPM cell lines. It was initially examined whether lung fibroblasts affect the migration of 

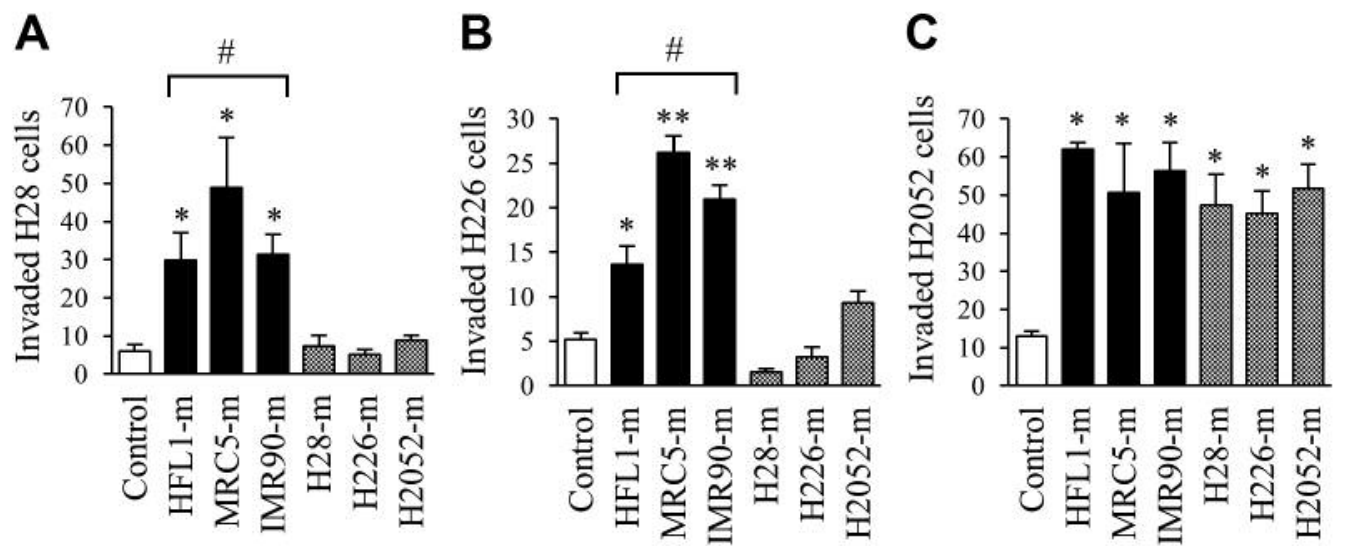

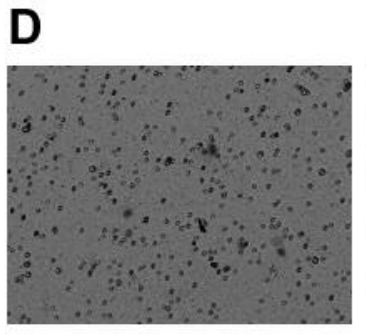

Control

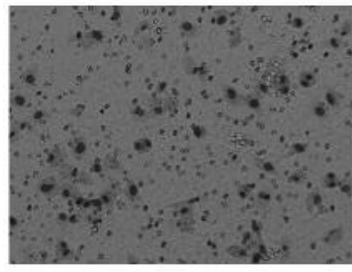

HFL1-m

Figure 2. Malignant pleural mesothelioma (MPM) cell invasion in response to lung fibroblast-derived media. Lung fibroblasts and MPM cells were cultured under serum-free conditions for 2 days, after which the culture media were collected. MPM cell invasion was assessed by Matrigel assay. (A) H28 cells; (B) H226 cells; (C) H2O52 cells. Data represent invaded cell number per field (mean \pm SEM of at least three independent experiments). ${ }^{*} p<0.05,{ }^{*} p<0.01$ compared to control (fresh medium). ${ }^{\#} p<0.05$ compared to a total of three MPM cell-derived media. (D) Representative images: H28 cell invasion in Matrigel assay.

MPM cell lines (H28, H226 and H2052). Three lung fibroblast types (HFL1, MRC5 and IMR90) were cultured in serum-free conditions and the cell culture media (HFL1-, MRC5 and IMR90-medium) were collected. When fibroblast-derived media were used as a chemoattractant, $\mathrm{H} 28$ cells exhibited significant migration at comparable rates between the three media types (HFL1-, MRC5- and IMR90-media) (Figure 1A). The response to MPM cell-derived media was also tested; H28-medium had no effect on H28 cell migration, while H226- and H2052-media enhanced the migration of H28 cells but to a lesser extent than fibroblast-derived media. The effect of fibroblast-derived media on MPM cell migration was also clear in H226 and H2052 MPM cells (Figure 1B and C); fibroblast-derived media markedly increased the migration of these MPM cells, while MPM-derived cell media had little effect. In the three MPM cell lines tested in this study, cell migration was statistically higher in response to a total of three lung fibroblast-derived media than to a total of three MPM cell-derived media. Figure 1D shows the migration of H28 cells in response to HFL1-derived medium as representative image of the observed effect.
To investigate whether lung fibroblasts affect the invasion of MPM cells, Matrigel invasion assays were performed using fibroblast- and MPM cell-derived media. The number of $\mathrm{H} 28$ cells that passed through the Matrigel-coated membrane clearly increased in response to each of the fibroblast-derived media, but not in response to the MPM cell-derived medium (Figure 2A). H226 cell invasion exhibited similar results to the $\mathrm{H} 28$ cells (Figure 2B). In H2052 cells, however, both fibroblast- and MPM cellderived media increased cell invasiveness (Figure 2C). Figure 2D illustrates H28 cell invasion in response to HFL1medium as a representative image of the observed effect.

Lung fibroblast-derived factors and their effects on MPM cell migration and invasion. Next, the factors produced by lung fibroblasts and the potential effects of these factors on the migration and invasion of MPM cells were examined. Fibronectin was shown to be released into the culture medium by fibroblasts $(4.5,3.6$ and $3.5 \mu \mathrm{g} / \mathrm{ml}$ in HFL-, MRC5- and IMR90-media, respectively) and to a lesser extent by $\mathrm{H} 2052$ cells $(1.2 \mu \mathrm{g} / \mathrm{ml}$ ) (Figure 3A). H28 and H226 cells, however, 

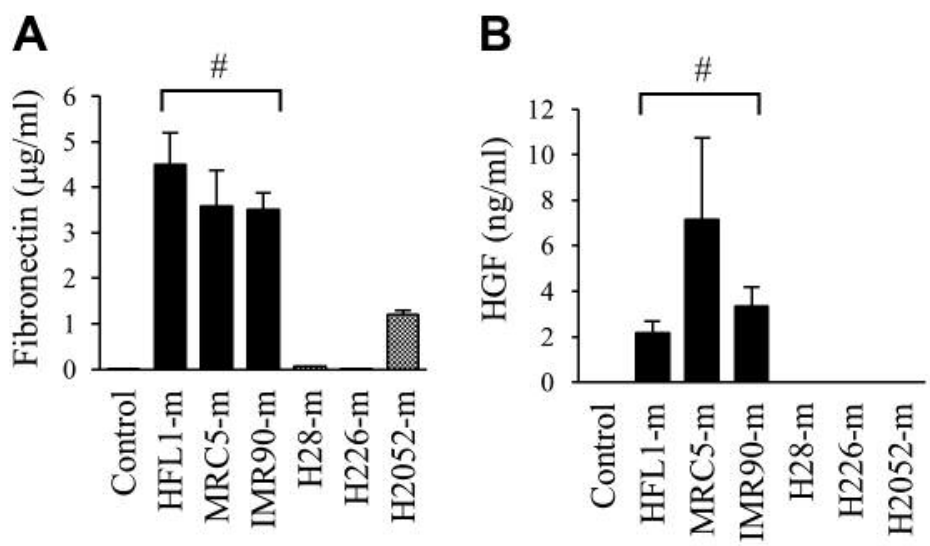

Figure 3. Fibronectin and hepatocyte growth factor (HGF) concentrations in cell-derived media. (A) Fibronectin and (B) HGF concentrations in cell-derived media were measured by ELISA. Data represent means \pm SEM of three independent experiments. ${ }^{*} p<0.05$ compared to a total of three MPM cell-derived media.
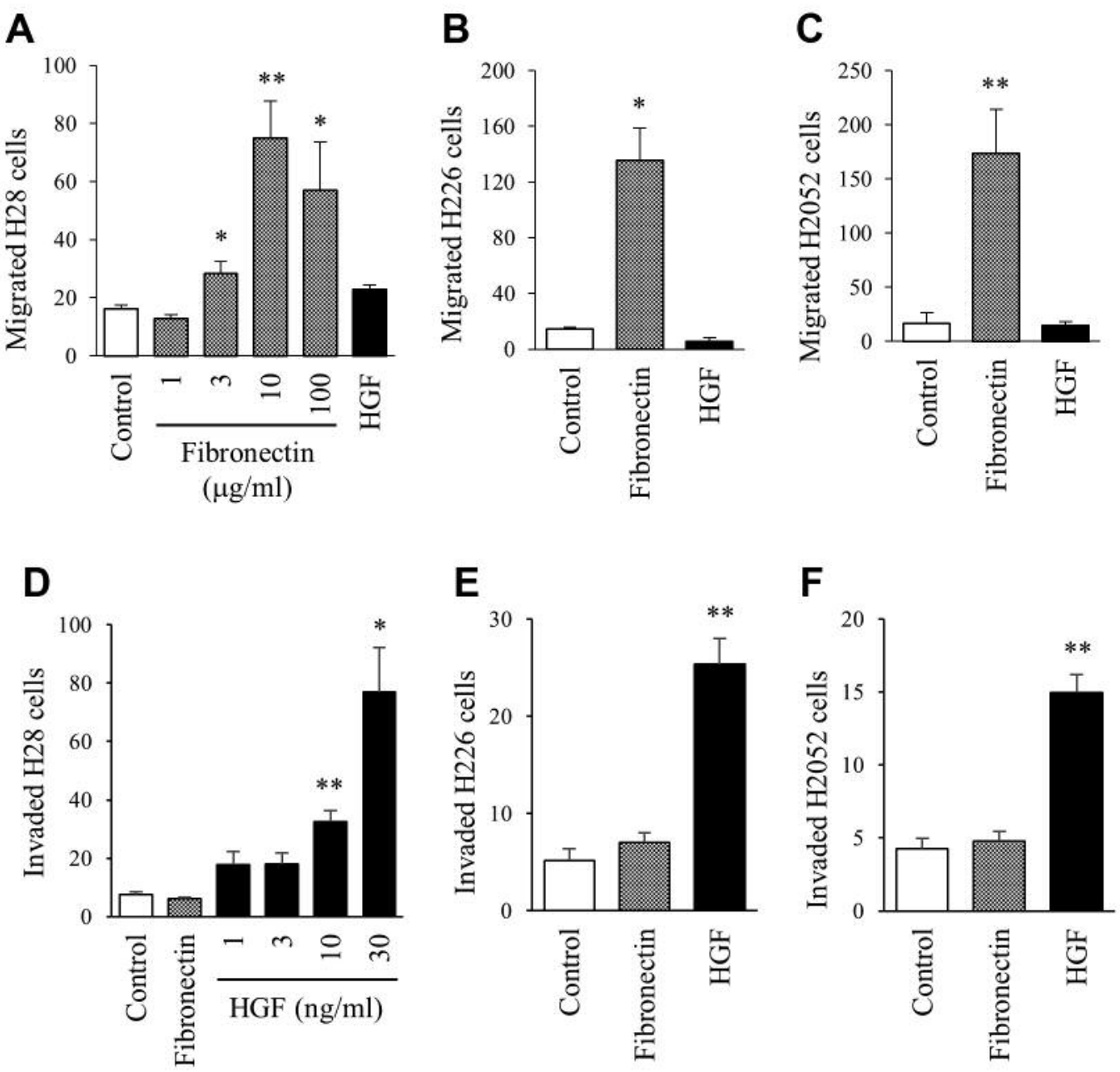

Figure 4. Effect of exogenous fibronectin and hepatocyte growth factor (HGF) on malignant pleural mesothelioma (MPM) cell migration and invasion. (A-C) MPM cell migration assessed by chemotaxis. Data represent migrated cell numbers per five fields (mean \pm SEM of four independent experiments). (D-F) invasion assessed by Matrigel assay. Data represent invaded cell numbers per field (mean \pm SEM of four independent experiments). ( $A$ and D) H28, ( $B$ and E) $\mathrm{H} 226$ and ( $C$ and F) $H 2052$ cells. Control, the absence of fibronectin and HGF; Fibronectin, $10 \mu g / m l$; HGF, $3 \mathrm{ng} / \mathrm{ml} .{ }^{*} p<0.05, * * p<0.01$ compared to control. 
A

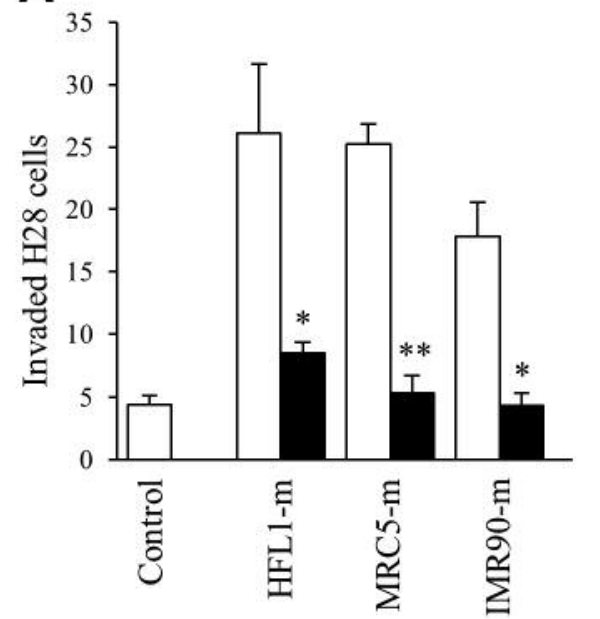

B

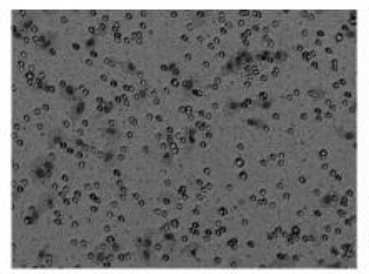

MRC5-m

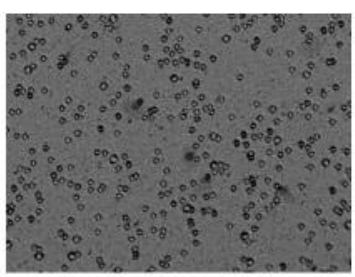

MRC5-m + anti-HGF Ab

Figure 5. Effect of anti-hepatocyte growth factor (anti-HGF) antibody (Ab) on fibroblast-derived media-enhanced H28 cell invasion. Anti-HGF antibody (neutralizing antibody) was added to fibroblast-derived media $1 \mathrm{~h}$ before Matrigel assay. (A) Matrigel assay. Data represent cell numbers per field (mean \pm SEM of three independent experiments). ${ }^{*} p<0.05$, **p<0.01 compared to the absence of anti-HGF antibody. (B) Representative images: $H 28$ cell invasion in Matrigel assay.

did not produce fibronectin. It was also shown that HGF was produced by the three fibroblasts cell types $(2.2,7.1$ and $3.4 \mathrm{ng} / \mathrm{ml}$ in HFL1-, MRC5- and IMR90-media, respectively), but not by MPM cells (Figure 3B); also, TGF- $\beta 1$ was detected in both fibroblast- and MPM cell-derived media at a variety of concentrations (data not shown). Epidermal growth factor was found to be absent in the media produced by fibroblasts and MPM cells (data not shown).

Exogenous fibronectin was shown to increase migration of the three MPM cell lines (Figure 4A-C). Generally, $3 \mu \mathrm{g} / \mathrm{ml}$ was found to be enough to increase migration significantly, while 10 $\mu \mathrm{g} / \mathrm{ml}$ yielded the greatest effect. HGF (up to $10 \mathrm{ng} / \mathrm{ml}$ ) did not affect migration of MPM cells. The migration of $\mathrm{H} 28$ cells was, furthermore, found to be unaffected by exogenous TGF- $\beta 1$ (data not shown).

In contrast to the chemotaxis results, the Matrigel invasion assay showed that exogenous fibronectin $(10 \mu \mathrm{g} / \mathrm{ml})$ had no effect on cell invasion (Figure 4D-F), while HGF was shown to increase invasion of $\mathrm{H} 28$ cells in a concentration-dependent manner at up to $30 \mathrm{ng} / \mathrm{ml}$ (Figure 4D). Cell invasion of $\mathrm{H} 226$ and $\mathrm{H} 2052$ cells was also shown to be enhanced by HGF (3 ng/ml), but not by fibronectin $(10 \mu \mathrm{g} / \mathrm{ml})$ (Figure $4 \mathrm{E}$ and $\mathrm{F}$ ).

Neutralizing anti-HGF antibody inhibits MPM cell migration and invasion enhanced by fibroblast-derived media. To confirm that the HGF present in fibroblast-derived media indeed functions to enhance MPM cell invasion, a neutralizing anti-HGF antibody was added into the fibroblast-derived media. As predicted, the fibroblast-derived media-induced enhancement of $\mathrm{H} 28$ cell invasion was inhibited by anti-HGF antibody (Figure 5). Similarly, antiHGF antibody partially inhibited the H226 cell invasion enhanced by fibroblast-derived media (data not shown).

MPM cell-medium stimulates the production of fibronectin and HGF by lung fibroblasts. To investigate whether the production of fibronectin and HGF by lung fibroblasts is affected by MPM cells, lung fibroblasts were cultured in MPM cell-derived media for 2 days, after which the culture media were collected. Interestingly, when cultured in MPM cell-derived media, lung fibroblasts produced fibronectin and HGF at higher levels than in control conditions (regular fibroblast-derived media) (Figure 6). Again, it must be noted that the fibronectin and HGF concentrations in the MPM cell-derived media were very low (Figure 3), except in the case of the fibronectin in H2052-medium.

\section{Discussion}

In the present study, we demonstrated that (i) lung fibroblast-derived medium enhances the migratory and invasive abilities of MPM cells via fibronectin and HGF production, respectively; and (ii) MPM cell-derived medium stimulates fibronectin and HGF production in lung 

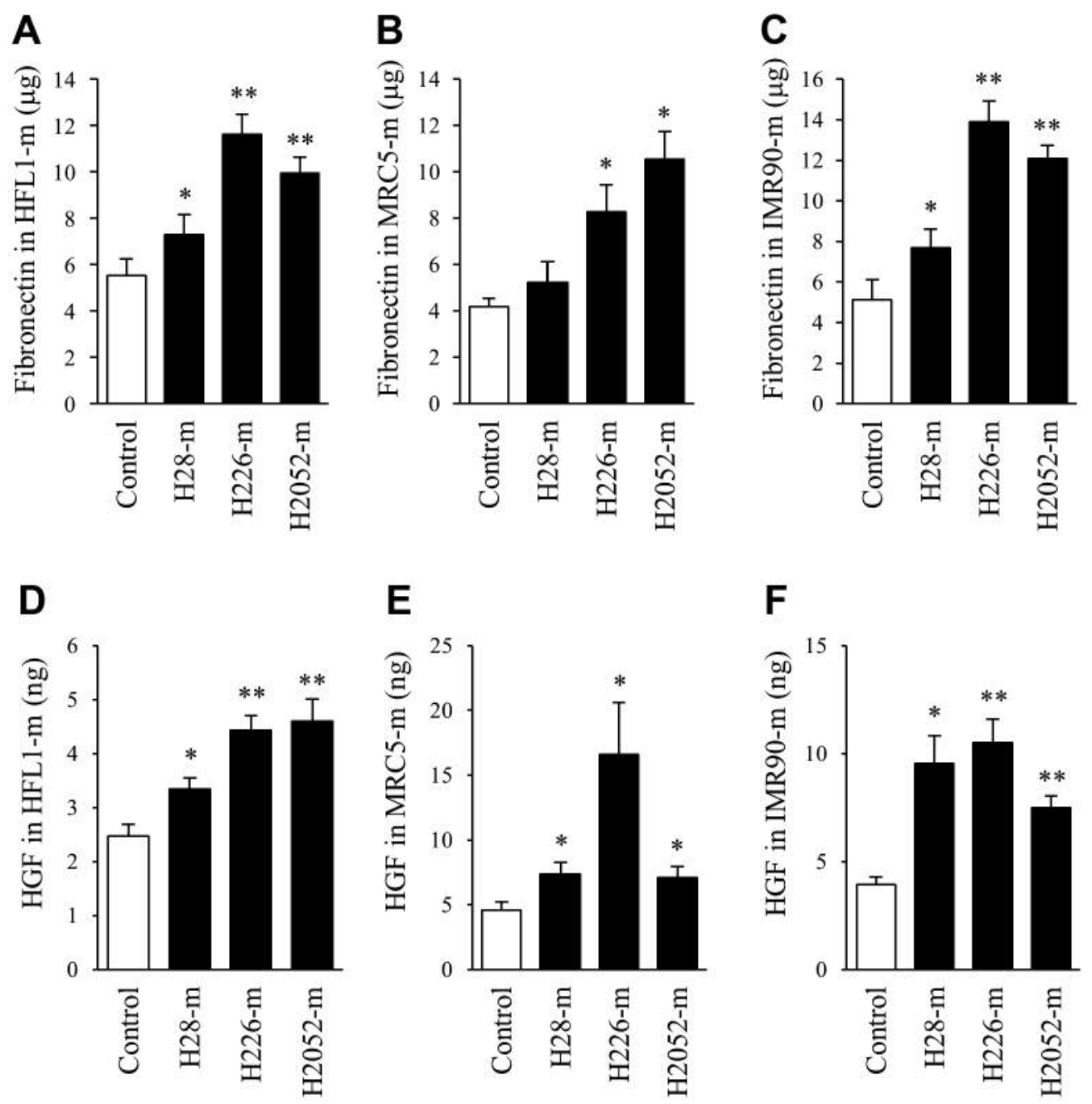

Figure 6. Production of fibronectin and hepatocyte growth factor $(H G F)$ from lung fibroblasts in response to malignant pleural mesothelioma (MPM) cell-derived media. Lung fibroblasts were cultured with control (fresh medium) or MPM cell-derived media for 2 days. (A-C) Fibronectin and (DF) HGF production (per million cells) were measured in fibroblast-derived media. Fibroblasts: ( $A$ and D) HFL1, (B and E) MRC5 and (C and F) IMR90 cells. Data represent mean \pm SEM of three independent experiments. ${ }^{*} p<0.05$, ${ }^{*} p<0.01$ compared to control.

fibroblasts. This interaction between fibroblasts and MPM cells is shown in Figure 7.

It is well-known that CAFs contribute to cancer progression $(6,9,10)$. Unlike "CAF", the term "TAF" has not yet permeated. The major reason for this is that most malignant tumors are cancers, that is, epithelial malignancies, while TAF refers to fibroblasts surrounding malignant epithelial and non-epithelial tumors. The term "TAF" is, therefore, used in a broader sense than "CAF". As in the case of CAFs, an important aspect of the concept of TAFs is their contribution to tumor progression. In a previous study, MPM cells were reported to stimulate TAFs to produce HGF (3). The current study expanded on this observation and showed a role for fibronectin and HGF production by fibroblasts in MPM cell migration and invasion, which, in turn, supports the concept of TAFs.

Fibronectin acts as a chemoattractant in many types of cells, including fibroblasts, vascular endothelial cells and cancer cells $(7,11,12)$. The present study shows that fibronectin functions as a chemoattractant for MPM cells, as well. Interestingly, however, fibronectin failed to promote MPM cell invasion through Matrigel-coated membranes. In contrast to fibronectin, HGF did augment invasion of MPM cells. Consistent with the findings reported here, it has been reported that HGF enhances MPM cell invasion through Matrigel (13). This effect may be associated with the stimulation of the expression of matrix metalloproteinases (MMPs), such as MMP-1 and MMP-9 (13). In addition, 


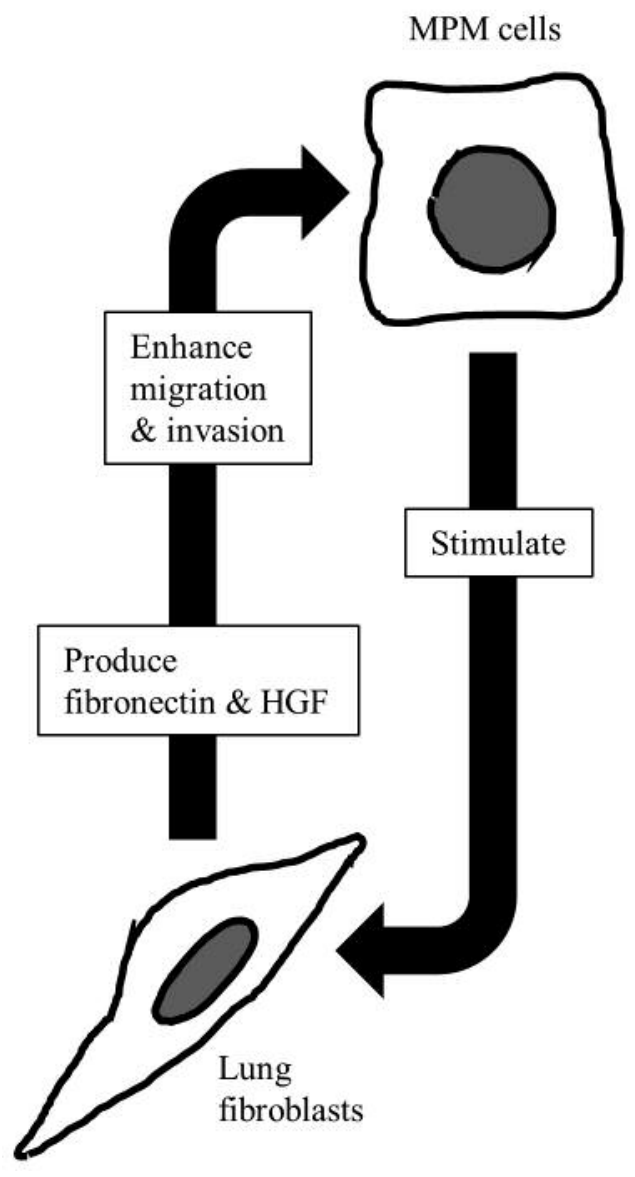

Figure 7. Schematic representation of the interaction between malignant pleural mesothelioma (MPM) cells and lung fibroblasts. Lung fibroblasts enhance MPM cell migration and invasion via fibronectin and hepatocyte growth factor (HGF) production. MPM cells stimulate these factors production in lung fibroblasts.

migration through the gelatin-coated membrane was also enhanced by HGF (13). These findings suggest that HGF seems to influence MPM cell invasion that requires some proteinase activity, including MMPs, but not on pure cell migration.

In the present study, anti-HGF antibody significantly inhibited MPM cell invasion enhanced by fibroblast-derived media, suggesting that the HGF produced from fibroblasts does indeed actively function to enhance MPM cell invasion. The concentration of HGF was previously shown to be measurable in malignant pleural effusions from patients with a wide variety of malignancies; the median concentration of HGF in pleural effusions obtained from three patients with MPM was $2.05 \mathrm{ng} / \mathrm{ml}$ (14), which is comparable to the HGF concentrations measured in fibroblast-derived media in the current study. The presence of HGF in pleural effusions is likely to play an important role in MPM progression.
Generally, the subpleural fibroblasts are thought to play a significant role in pleural fibrosis; however, increasing evidence suggests that mesothelial cells also play a role in pleural fibrosis (15). Management of TAFs may decrease HGF concentrations in pleural effusion and, thus, lead to the inhibition of MPM progression.

HGF functions by binding to the mesenchymalepithelial transition (MET) receptor and inhibition of the MET receptor has been shown to suppress MPM cell growth and migration $(2,16)$. Moreover, it has been reported that plasma cell membrane localization of c-MET is associated with longer overall survival (25 vs. 13 months) in patients with MPM (17) and the HGF-MET signaling pathway may, therefore, represent a potential target for the treatment of MPM.

In conclusion, the findings reported here provide additional evidence for an interaction between MPM cells and fibroblasts that plays a role in MPM progression. Fibronectin and HGF were shown to be produced by lung fibroblasts and, furthermore, to enhance MPM cell migration and invasion. The production of these factors by lung fibroblasts was stimulated by MPM cells. These findings contribute to the development of anti-TAF therapeutic strategies.

\section{Acknowledgements}

The Authors thank Ms. Takimi Tamaki for the excellent experimental support. The manuscript was edited by Editage, an English proofreading company.

\section{References}

1 Saint-Pierre MD, Pease C, Mithoowani H, Zhang T, Nicholas GA, Laurie SA and Wheatley-Price P: Malignant pleural mesothelioma outcomes in the era of combined platinum and folate antimetabolite chemotherapy. Lung Cancer Int 2015: 590148, 2015.

2 Suzuki Y, Sakai K, Ueki J, Xu Q, Nakamura T, Shimada H, Nakamura $\mathrm{T}$ and Matsumoto K: Inhibition of Met/HGF receptor and angiogenesis by NK4 leads to suppression of tumor growth and migration in malignant pleural mesothelioma. Int $\mathbf{J}$ Cancer 127(8): 1948-1957, 2010.

3 Li Q, Wang W, Yamada T, Matsumoto K, Sakai K, Bando Y, Uehara H, Nishioka Y, Sone S, Iwakiri S, Itoi K, Utsugi T, Yasumoto K and Yano S: Pleural mesothelioma instigates tumorassociated fibroblasts to promote progression via a malignant cytokine network. Am J Pathol 179(3): 1483-1493, 2011.

4 Vogelzang NJ, Rusthoven JJ, Symanowski J, Denham C, Kaukel E, Ruffie P, Gatzemeier U, Boyer M, Emri S, Manegold C, Niyikiza $\mathrm{C}$ and Paoletti P: Phase III study of pemetrexed in combination with cisplatin versus cisplatin alone in patients with malignant pleural mesothelioma. J Clin Oncol 21(14): 26362644, 2003.

5 Majety M, Pradel LP, Gies M and Ries CH: fibroblasts influence survival and therapeutic response in a $3 \mathrm{D}$ co-culture model. PLoS One 10(6): e0127948, 2015. 
6 Bremnes RM, Dønnem T, Al-Saad S, Al-Shibli K, Andersen S, Sirera R, Camps C, Marinez I and Busund LT: The role of tumor stroma in cancer progression and prognosis: Emphasis on carcinoma-associated fibroblasts and non-small cell lung cancer. J Thorac Oncol 6(1): 209-217, 2011.

7 Kanaji N, Basma H, Nelson A, Farid M, Sato T, Nakanishi M, Wang X, Michalski J, Li Y, Gunji Y, Feghali-Bostwick C, Liu X and Rennard SI: Fibroblasts that resist cigarette smoke-induced senescence acquire profibrotic phenotypes. Am J Physiol Lung Cell Mol Physiol 307(5): L364-L373, 2014.

8 Kanaji N, Bandoh S, Ishii T, Fujita J, Ishida T, Matsunaga T and Kubo A: Cytokeratins negatively regulate the invasive potential of lung cancer cell lines. Oncol Rep 26(4): 763-768, 2011.

9 Navab R, Strumpf D, Bandarchi B, Zhu CQ, Pintilie M, Ramnarine VR, Ibrahimov E, Radulovich N, Leung L, Barczyk M, Panchal D, To C, Yun JJ, Der S, Shepherd FA, Jurisica I and Tsao MS: Prognostic gene-expression signature of carcinomaassociated fibroblasts in non-small cell lung cancer. Proc Natl Acad Sci USA 108(17): 7160-7165, 2011.

10 Kim SH, Choe C, Shin YS, Jeon MJ, Choi SJ, Lee J, Bae GY, Cha HJ and Kim J: Human lung cancer-associated fibroblasts enhance motility of non-small cell lung cancer cells in coculture. Anticancer Res 33(5): 2001-2009, 2013.

11 Kanaji N, Sato T, Nelson A, Wang X, Li Y, Kim M, Nakanishi M, Basma H, Michalski J, Farid M, Chandler M, Pease W, Patil A, Rennard SI and Liu X: Inflammatory cytokines regulate endothelial cell survival and tissue repair functions via NF- $x \mathrm{~B}$ signaling. J Inflamm Res 4: 127-138, 2011.

12 Geng L, Ali SA, Marshall JF, Mackay CL, Hart IR, Delcommence M, Streuli CH and Rees RC: Fibronectin is chemotactic for CT 26 colon carcinoma cells: sub-lines selected for increased chemotaxis to fibronectin display decreased tumorigenicity and lung colonization. Clin Exp Metastasis 16(8): 683-691, 1998.
13 Harvey P, Clark IM, Jaurand MC, Warn RM and Edwards DR: Hepatocyte growth factor/scatter factor enhances the invasion of mesothelioma cell lines and the expression of matrix metalloproteinases. Br J Cancer 83(9): 1147-1153, 2000.

14 Eagles G, Warn A, Ball RY, Baillie-Johnson H, Arakaki N, Daikuhara Y and Warn RM: Hepatocyte growth factor/scatter factor is present in most pleural effusion fluids from cancer patients. Br J Cancer 73(3): 377-381, 1996.

15 Mutsaers SE, Prele CM, Brody AR and Idell S: Pathogenesis of pleural fibrosis. Respirology 9(4): 428-440, 2004.

16 Mukohara T, Civiello G, Davis IJ, Taffaro ML, Christensen J, Fisher DE, Johnson BE and Jänne PA: Inhibition of the met receptor in mesothelioma. Clin Cancer Res 11(22): 8122-8130, 2005.

17 Levallet G, Vaisse-Lesteven M, Le Stang N, Ilg AG, Brochard P, Astoul P, Pairon JC, Bergot E, Zalcman G and Galateau-Sallé F: Plasma cell membrane localization of c-MET predicts longer survival in patients with malignant mesothelioma: A series of 157 cases from the MESOPATH Group. J Thorac Oncol 7(3): 599-606, 2012 